\title{
Development of simulator for microgrid system
}

\author{
H H Kim, H S Tak, H G Cho, H J Kim* \\ Dept. of Electrical and Computer Engineering, Pusan National University, Busan, 609735, Republic of Korea
}

\begin{abstract}
This study proposes and develops a simulator for the prediction and control experiments of a microgrid system. A microgrid is a next-generation intelligent power grid that optimizes energy efficiency by combining information technology with a power grid. The microgrid needs to efficiently distribute power to both producers and consumers and therefore, requires an optimized grid control system that predicts power generation and consumption. The existing power distribution simulator is inconvenient to use if the user does not possess electrical knowledge. In this paper, we propose a simulator for the power prediction and control experiments of a microgrid. The proposed system uses past data or scenarios rather than electrical knowledge for the simulation. In addition, it provides an intuitive graphical interface that is developed as a web page considering user accessibility requirements. Finally, the monitoring function is explained by way of examples in order to confirm the simulation results.
\end{abstract}

Keywords: Microgrid, Simulator

\section{Introduction}

A microgrid has been proposed as a next generation power grid owing to power demand increases, environmental pollution and resource depletion problems. The microgrid is an 'intelligent power grid' that connects producers and consumers in a bidirectional manner using information technology to manage unidirectional power networks. It aims to operate the power system efficiently based on the power plant, transmission and distribution facilities, and power consumer information [1].

In the microgrid, suppliers need to be able to adjust their supply by identifying the power usage status, and consumers should be able to control their usage time and usage of electronic devices. Unlike the existing power grid, the use of renewable energy with irregular power generation has increased as the system has been converted to a distributed power system. As a result, the microgrid is being promoted as a next-generation power grid business in many countries. Korea is also developing microgrid demonstration complexes in Jeju Island and conducting research on their internal facilities and power grid operation [2].

The microgrid requires a control system for efficient power usage that can predict and respond to power demand. However, existing simulators not only require electrical knowledge of the modules to be configured, but are also limited to simultaneous prediction and control. In this paper, we propose a simulator for the grid configuration, power estimation, and operation of a microgrid system. The proposed simulator excludes the operation principle of the constituent equipment as much as possible and provides an environment that enables various experiments to be conducted using past data and scenarios. To explain this, we introduce the configuration and interface of the simulator, and demonstrate the implementation results through a monitoring example.

\footnotetext{
* Manuscript received Jan. 23, 2018; revised June 5, 2018.

Corresponding author. Tel.: +82-51-510-2871; E-mail address: quant1216@pusan.ac.kr.

doi: $10.12720 /$ sgce.7.3.195-200
} 


\section{Related Work}

The microgrid improves reliability, efficiency, and safety by combining information and communication technology with existing power grids. In addition, various studies have been conducted to optimize energy efficiency. It is expected to provide high quality energy and various additional services through bidirectional power information exchange [3].

Currently, research on Microgrid software is mainly focused on IoT based mutual network configuration and microgrid security research. However, there is little research on efficient use of microgrid system through design and control. In Europe, electricity is supplied by private companies, and microgrid simulators designed to reduce electricity bills are being designed and applied [4]. In Korea, a power control simulator model considering mechanical characteristics is proposed [5].

When a microgrid is constructed, both power producers and consumers have problems with efficient power use. In the case of producers, there is a problem of minimizing surplus power while preventing blackout, and consumers want to use tariffs for external power by using self-produced renewable energy. In addition, if the power resources and the control modules do not operate efficiently in the microgrid system, the stability of the power grid will be adversely affected. The Microgrid requires a control system for efficient power usage that anticipates and responds to power demands sensitive to the surrounding environment while solving the preceding problems. However, existing power distribution simulators require electrical knowledge related to the constructed transmission and distribution system and power generation, and most of them provide only a part of prediction and control functions. Table 1 compares simulators for power distribution in the conventional electric field.

Table 1. Comparison of power distribution simulators.

\begin{tabular}{|c|c|c|c|c|}
\hline System Name & Parameter Setting & Open Source & Interface & Monitoring \\
\hline GridLAB-D [6] & $\mathrm{O}$ & $\mathrm{O}$ & CLI & $\mathrm{X}$ \\
\hline OpenDSS & $\mathrm{O}$ & $\mathrm{O}$ & CLI & $\mathrm{X}$ \\
\hline Simulink & $\mathrm{O}$ & $\mathrm{X}$ & GUI & $\mathrm{O}$ \\
\hline MapleSim [9] & $\mathrm{O}$ & $\mathrm{X}$ & GUI & $\mathrm{O}$ \\
\hline
\end{tabular}

\section{Microgrid Optimal Operation Simulator}

\subsection{System overview}

In this paper, we propose a simulator that can perform microgrid configuration, power distribution and machine operation with minimal electrical knowledge. The proposed system seeks to minimize the operation principle of the equipment as much as possible and provides an environment that enables various experiments to be conducted using past data and scenarios. In addition, we design simulators that emphasize monitoring functions for experiments in prediction and control systems, so that we can easily design and conduct experiments on microgrid system.

Existing power distribution simulators require expert knowledge of electricity and conversion of electrical characteristics such as frequency, voltage, DC and AC. However, it is possible to internalize basic conversion process and to analyze power demand forecasts and the power situation using power data. Therefore, the proposed system performs the simulation of the configured grid with the information generated by the facilities.

Microgrid facilities are largely divided into stand-alone type and grid-connected type. Stand-alone type is a system that operates all facilities using independent electric production system without connection of power plants. On the other hand, the grid-connected type is a power generation facility capable of flexibly controlling the use and overload of electric power in connection with a power plant. In the case of standalone type, there is a problem that the power is not available when the system is turned off due to a failure of some machines or when power is low. The simulator proposed in this paper allows users to freely select the external power supply when constructing the grid, and it is possible to perform a comparative 
experiment on two facilities.

\subsection{System interface}

The simulator is developed as a web-based open source program for users to access and operate easily. Users can design and simulate a microgrid system by accessing the program from anywhere on the Internet. We have also developed the graphical interface such that users can intuitively understand and use the software. Fig. 1 displays the entire simulator program developed in this study.

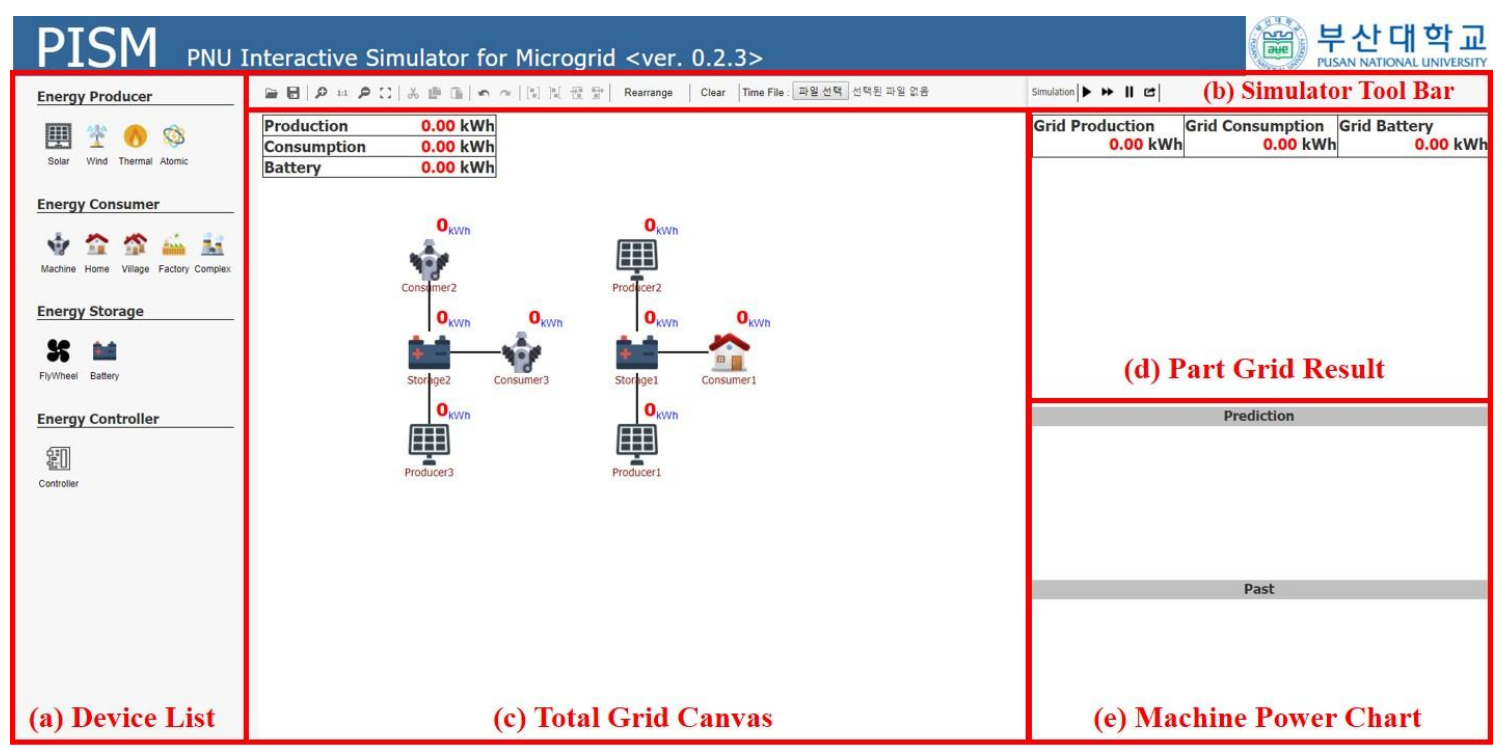

Fig. 1. Simulation System Main Screen.

The details of each part of the interface are as follows.

Table 2. Specification device list (Figure 1-(a)).

\begin{tabular}{ll}
\hline Device Type & Devices \\
\hline Power Generation device & Solar Power, Wind Power, Thermal Power, Atomic Power \\
Power Consumption device & Machine, Home, Village, Factory, Complex \\
Power Storage device & Battery \\
Power Controller & Controller \\
\hline
\end{tabular}

Table 2 shows the specification of Device List. Device List represents power devices that can be used in grid designing. Power devices are divided according to their function and then divided according to the usage, volume and property of each mechanism. Energy Producer part consists of two renewable energy generators and two power plants. The difference is that the renewable energy generators are not constant for power generation and sometimes power shortages can occur. But power plants do not cause power shortages. Energy Consumer part consists of five examples because power consumption and power consumption pattern are different for different users. Machine is just for general use. The difference between Home and Village is power consumption because a village consumes more power than a home. The difference between Factory and Complex is the same as the difference between Home and Village. And the difference between Home, Village and Factory, Complex is power consumption patterns because Home, Village usually use power at night, but Factory, Complex use power during a day. Energy Storage part has a general-purpose battery (lithium-ion battery) and Energy Controller part has a controller. 
Table 3. Specification simulator tool bar (Figure 1-(b)).

\begin{tabular}{ll}
\hline Tool Type & Tools \\
\hline File & Open, Save \\
Zoom & Zoom in, Zoom to original size, Zoom out, Fit content \\
Edit & Cut, Copy, Paste \\
Cancel & Undo, Redo \\
Group & Group, Ungroup, Enter group, Leave group \\
Rearrange & Apply orthogonal layout to grid \\
Clear & Grid clear \\
Time & Time file selection \\
Simulation & One step simulation, Simulation play, Simulation pause, Export result \\
\hline
\end{tabular}

Table 3 shows the specification of Simulator Tool Bar. Simulator Tool Bar represents the tools which is needed for designing the grid and proceeding with the simulation. Time file selection button is used to input a time unit file to set time environment of simulation. Except for this, the function of each button is easily known by name.

Table 4. Action \& event of total grid canvas (Figure 1-(c)).

\begin{tabular}{ll}
\hline Action & Event \\
\hline Left click in space & Create a node(machine) \\
Left click created machine & Draw part grid and data chart of clicked machine \\
Left double click created machine & Change the label \\
Right click created machine & Pop up a data selection window \\
Mouse hover to producer machine & Show the temperature and irradiation chart if they are set \\
Dragging with node selection & Move the machine \\
Dragging without node selection & Create an edge(connection) \\
Wheel up & Zoom in \\
Wheel down & Zoom out \\
\hline
\end{tabular}

Table 4 shows the actions that are used in Total Grid Canvas for designing grid. A user can design the grid system by clicking on one of a device in Device List and then clicking on the Total Grid Canvas. And a user can connect the machines to each other by dragging. Once the past power production or consumption power data file is selected to each device, then the simulation can be performed by predicting the power production or consumption.

Table 5. Symbol \& meaning of part grid result (Figure 1-(d)).

\begin{tabular}{ll}
\hline Device Type & Symbol(Meaning) \\
\hline Power Generation device & Work(blue), Not Work(red) \\
Power Consumption device & Work(blue), Not Work(red) \\
Power Storage device & Charge(+), Discharge(-), Full(F), Empty(E), Stable(S) \\
\hline
\end{tabular}

Table 5 shows the symbols and meanings of the simulation progress of selected grid. Power production devices and power consumption devices use color to indicate whether the machine is operating. The power storage devices indicate whether the machine is operating using characters. An example of this is shown in the next chapter, grid monitoring.

\subsection{Grid Monitoring}

This simulator is designed to monitor the simulation situation on a time basis. The simulator allows 
you to monitor the power status of the entire grid, the partial grid, and the machine. Fig. 2 is an excerpt of the monitoring function part of the overall system during simulation. Fig. 2-(a) shows the power status of the entire grid, the configuration of the grid, and the current power status of each machine. In this example, the total production, total consumption and total storage are $17.35 \mathrm{kWh}, 138.80 \mathrm{kWh}$ and $2733.47 \mathrm{kWh}$, respectively. When you click on one of the machines in Total Grid Canvas, Part Grid Result and Machine Power Chart of the clicked machine come out. Fig. 2-(b) shows the power status and machine operation of the selected partial grid. In this example, Grid production, grid consumption and grid battery are $7.47 \mathrm{kWh}, 48.52 \mathrm{kWh}$, and $871.29 \mathrm{kWh}$, respectively. Also you can know information such as "the Producer 3 is working", "the Consumer 2, 3 is working" and "the Storage 2 is discharging". Fig. 2-(c) shows the power status flow of selected machine. The above chart shows the flow of the power status after the simulation has been carried out, and the chart below shows the flow of power status of the input data.

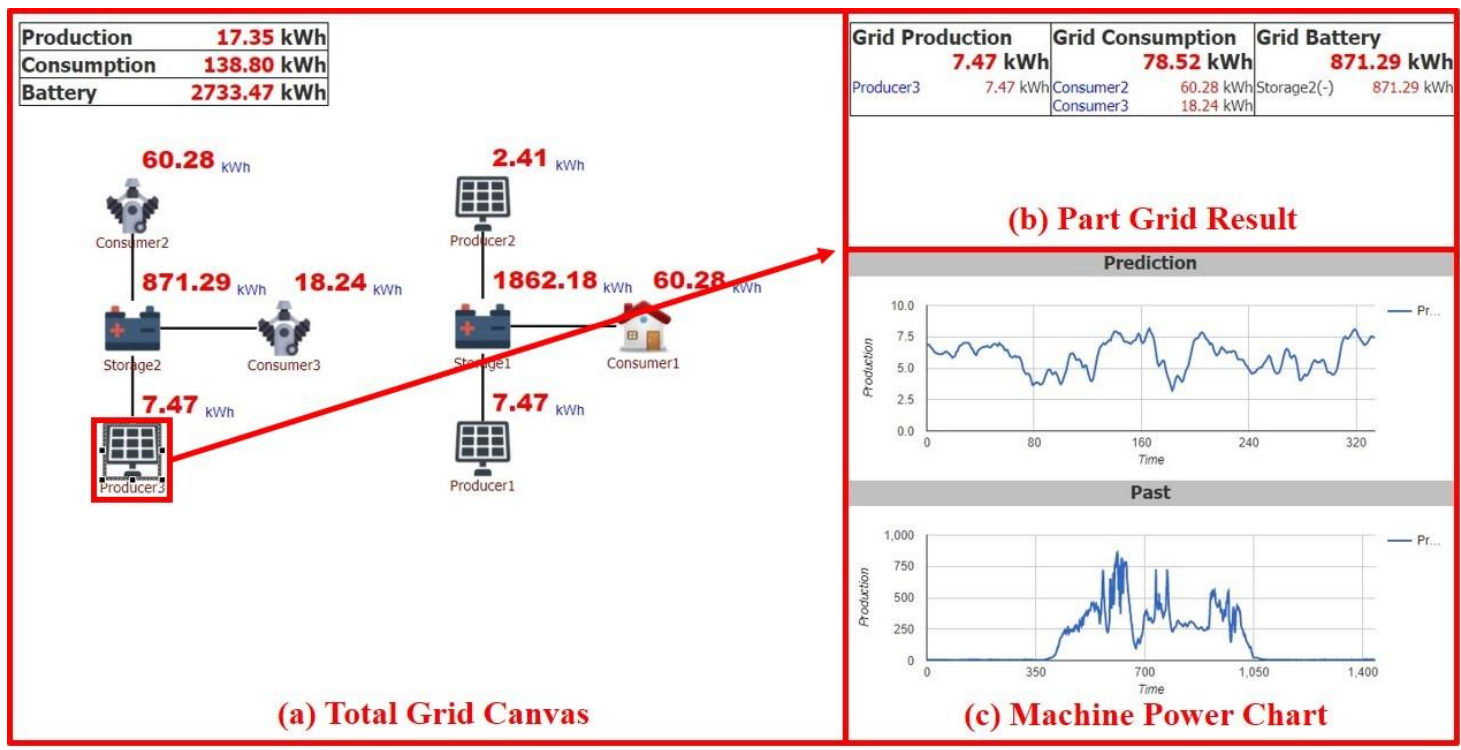

Fig. 2. Monitoring functions of simulator when Producer 3 is selected.

\section{Conclusion and Future Work}

In this study, we proposed and developed a base simulator specialized in the design and experimentation of the prediction and control system of a microgrid. In order to solve the problem of the configuration complexity of existing simulators, the proposed simulator proceeds using past and predictive data. As a result, various experiments can be easily conducted.

As part of a future study, we will conduct research for optimizing a microgrid by applying various power prediction algorithms. Through information exchange between storage devices, we intend to develop controllers that can resolve power outage problems while minimizing power plant intervention. In addition, controllers should take into account the characteristics of a machine, such as preventing the complete charge or discharge of the machine. Furthermore, we intend to improve the prediction accuracy of each device by considering surrounding information and other parameters, rather than only the prediction of a device's power data.

\section{Acknowledgement}

This research was supported by Basic Research Laboratory through the National Research Foundations of Korea funded by the Ministry of Science, ICT and Future Planning (NRF-2015R1A4A1041584). 


\section{References}

[1] Kim D. Smart grid concept. Bulletin of the Society of Naval Architects of Korea, 2013: 50(1):45-49.

[2] Lee M. Analysis of smart grid technologies and domestic and foreign policy trends. Journal of Korean Institute of Information Technology, 2013: 11(8):181-187.

[3] Sung D, Song N, Go G, Cha J, Bae G, Jang H. Convergence of power system technology and information communication technology in smart grid. Communications of the Korea Information Science Society, 2013: 31(3):10-21.

[4] Ursachi A, Bordeasu D. Smart grid simulator. International Journal of Civil, Architectural, Structural and Construction Engineering, 2014: 8(5):519-522.

[5] Jo J, Park S, Lee D, Jo Y, Jun M. Integrated simulation model for designing distributed energy management system in microgrid system. Journal of The Korean Institute of Intelligent Systems, 2011: 21(1):29-35.

[6] Chassin DP, Schneider K, Gerkensmeyer C. GridLAB-D:An open-source power systems modeling and simulation environment. Transmission and Distribution Conference and Exposition, 2008: 1-5.

[7] Montenegro D, Hernandez M, Ramos GA. Real time OpenDSS framework for distribution systems simulation and analysis. Transmission and Distribution: Latin America Conference and Exposition (T\&D-LA), 2012 Sixth IEEE/PES, 2012; 1-5.

[8] Logue D, Krein PT. Simulation of electric machinery and power electronics interfacing using MATLAB/SIMULINK. Computers in Power Electronics, 2000; 34-39.

[9] Hřebíček J, Řezáč M. Modelling with maple and maplesim. 22nd European Conference on Modelling nad Simulation ECMS 2008 Proceedings, 2008; 60-66. 